\title{
À propos des Chrysomelidae de l'archipel de Madère : nouvelles données faunistiques (Coleoptera)
}

\author{
Jean-David Chapelin-VIscardi ${ }^{1}$, Philippe Ponel $^{2} \&$ Serge Doguet* \\ ${ }^{1}$ Laboratoire d'Éco-Entomologie, 5 rue Antoine-Mariotte, F - 45000 Orléans \\ $<$ chapelinviscardi@laboratoireecoentomologie.com> \\ ${ }^{2}$ Institut Méditerranéen de Biodiversité et d'Écologie marine et continentale (IMBE), Aix-Marseille Université, \\ Univ. Avignon, CNRS, IRD, Technopôle de l'Environnement Arbois-Méditerranée, BP 80, \\ F-13545 Aix-en-Provence cedex 04 <philippe.ponel@imbe.fr>
}

(Accepté le 2.V.2018; publié le 22.VI.2018)
Résumé. - Des données sur des Coléoptères Chrysomèles de l'archipel de Madère sont apportées. Quatre espèces méritent d'être mises en exergue, dont Longitarsus melanocephalus (Degeer, 1775) qui est signalée pour la première fois de l'archipel.

\begin{abstract}
About the Chrysomelidae of Madeira archipelago: new faunistic data (Coleoptera). Additional data about Chrysomelidae of Madeira archipelago are provided. Four species deserve to be highlighted, especially Longitarsus melanocephalus (Degeer, 1775), which is reported for the first time from these islands.
\end{abstract}

Keywords. - Leaf beetles, Madeira archipelago, biodiversity, first record, faunistics.

La faune de Chrysomélides de l'archipel de Madère a fait l'objet d'assez nombreuses publications, citons par exemple Erber (1984a, 1984b, 1986), Erber \& Hinterseher (1988), Erber \& Franquinho Aguiar (1996), Jolivet (2007), Borges et al. (2008), et semblait relativement bien connue. En excluant les Bruchinae, 41 espèces de Chrysomélides sont répertoriées sur ces îles (Borges et al., 2008). Deux courts séjours réalisés dans l'archipel par les deux premiers auteurs de cette note ont permis de collecter un certain nombre d'espèces intéressantes ou nouvelles pour la dition. Nous pensons utile de résumer ici les résultats obtenus au cours de ces prospections.

\section{MatéRIELS et MÉTHOdeS}

Le premier séjour a été effectué à Madère même en 2001 par le second auteur, en compagnie de Peter Stüben, spécialiste des Charançons et particulièrement des Cryptorhynchinae, Insectes pour la plupart saproxylophages. Pour cette raison, divers secteurs de vieille laurisylve de l'île ont été prospectés en priorité. Le second séjour, réalisé en 2015 (JDCV), a permis de visiter la moitié est de l'île de Madère ainsi que l'île de Porto Santo.

Les prospections entomologiques ont été réalisées au moyen des techniques traditionnelles : prélèvements à vue, fauchage et battage. L'ensemble du matériel a été étudié au retour de voyage et l'un de nous (SD) a notamment pu étudier ou contrôler les spécimens d'identification délicate et les espèces remarquables.

Les sites et les dates de relevés sont précisés dans la tableau I. Afin de simplifier la présentation des données, celles-ci seront par la suite indiquées par un numéro.

\footnotetext{
* Nous avons souhaité associer Serge Doguet à ce petit travail, en témoignage d'amitié, et de reconnaissance pour son aide inlassable, ses encouragements et son enthousiasme. C'est pour discuter de ce projet de note sur les Chrysomélides de Madère et des identifications qu'il venait juste de terminer que Serge avait tenu à venir spécialement à la gare de Lyon, à Paris, le 21 décembre 2015, pour rencontrer le second auteur avant son retour vers Aix-en-Provence, alors que la maladie rendait ses déplacements déjà très difficiles et douloureux. Ce fut leur dernière rencontre avant que ses forces déclinantes ne le contraignent à entrer en clinique.
} 
Tableau I. - Stations prospectées (lieux, coordonnées et dates).

\begin{tabular}{|c|l|l|c|c|c|l|}
\hline $\begin{array}{c}\mathbf{N}^{\circ} \\
\text { relevé }\end{array}$ & Île & Commune ou lieu-dit & Latitude & Longitude & Date & \multicolumn{1}{|c|}{ Précisions } \\
\hline 1 & Madère & Boca da Encumeada & 32,754166 & $-17,018611$ & 7. VII.2001 & Laurisylve $(993 \mathrm{~m})$ \\
\hline 2 & Madère & Encumeada, Levada do Norte & 32,753611 & $-17,036388$ & 8. VII.2001 & Laurisylve $(913 \mathrm{~m})$ \\
\hline 3 & Madère & $\begin{array}{l}\text { Entre Camara de Lobos et } \\
\text { Cabo Girão }\end{array}$ & 32,656324 & $-16,990949$ & 29. III.2015 & Divers milieux \\
\hline 4 & Madère & Fanal, Casa do Guarda Florestal & 32,809444 & $-17,141111$ & 13. VII.2001 & Laurisylve $(1161 \mathrm{~m})$ \\
\hline 5 & Madère & Funchal, Jardim Botanico & 32,661944 & $-16,895555$ & 7. VII.2001 & Jardin $(295 \mathrm{~m})$ \\
\hline 6 & Madère & Ponta do São Lourenço & 32,740277 & $-16,704722$ & 11. VII.2001 & Pelouses xériques \\
\hline 7 & Madère & Ponta do São Lourenço & 32,744576 & $-16,700676$ & 29. III.2015 & Pelouses xériques \\
\hline 8 & Madère & $\begin{array}{l}\text { Porto Moniz, Pombais, } \\
\text { Ribeira do Tristão }\end{array}$ & 32,856666 & $-17,205555$ & 10. VII.2001 & Ravin $(177 \mathrm{~m})$ \\
\hline 9 & Madère & São Jorge & 32,829166 & $-16,897778$ & 9. VII.2001 & Falaise littorale $(18 \mathrm{~m})$ \\
\hline 10 & Madère & Sud de Santana & 32,793798 & $-16,883725$ & 1. IV.2015 & $\begin{array}{l}\text { Friche de bord de } \\
\text { route }(580 \mathrm{~m})\end{array}$ \\
\hline 11 & Porto Santo & Au nord du Pico Castelo & 33,083474 & $-16,332549$ & 4. IV.2015 & $\begin{array}{l}\text { Zone forestière } \\
(\text { environ } 300 \mathrm{~m})\end{array}$ \\
\hline 12 & Porto Santo & Au sud du Pico do Facho & 33,084018 & $-16,324203$ & 4. IV.2015 & Alt. $\approx 450 \mathrm{~m}$ \\
\hline 13 & Porto Santo & Sous le Pico do Ana Ferreira & 33,044247 & $-16,376268$ & 3. IV.2015 & Alt. $\approx 200 \mathrm{~m}$ \\
\hline 14 & Porto Santo & $\begin{array}{l}\text { De Cambo do Baixo à Praia } \\
\text { de Zimbralinho }\end{array}$ & 33,037519 & $-16,376268$ & 3. IV.2015 & Alt. $\approx 75 \mathrm{~m}$ \\
\hline 15 & Porto Santo & Vila Baleira & 33,056926 & $-16,336538$ & 3. IV.2015 & Dunes littorales \\
\hline
\end{tabular}

\section{RÉSULTATS}

Les espèces recensées, ainsi que les informations relatives à leur observation sont les suivantes.

\section{Sous-famille Alticinae}

Longitarsus cerinthes (Schrank, 1798). - Relevé n³, 1 ex.; relevé nº ${ }^{\circ}, 1$ ex.

Longitarsus cinerariae Wollaston, 1854 [syn. L. consanguineus] (fig. 1). - Relevé ${ }^{\circ} 1,5$ ex.

Longitarsus isoplexidis Wollaston, 1854 [syn. L. masoni] (fig. 2). - Relevé $n^{\circ} 5,1$ ex. ; relevé ${ }^{\circ} 9,4$ ex.

Longitarsus melanocephalus (Degeer, 1775). - Relevé $n^{\circ} 10,8$ ex.

Longitarsus ochroleucus lindbergi Madar \& Madar, 1963. - Relevé n¹1, 6 ex.; relevé n¹2, 7 ex. sur Rapistrum rugosum (L.) All ; relevé ${ }^{\circ} 14,1$ ex.; relevé n 15,1 ex.

Mniophilosoma laeve Wollaston, 1854 (fig. 3). - Relevé $n^{\circ} 2,2$ ex.; relevé $n^{\circ} 4,1$ ex.

Ochrosis ventralis (Illiger, 1807). - Relevé nº, 1 ex., battage de Ficus; relevé n 11,1 ex. fauchage de Poacées; relevé n 13,1 ex.

Phyllotreta consobrina (Curtis, 1837). - Relevé $\mathrm{n}^{\circ} 10,1$ ex.

Psylliodes hospes Wollaston, 1854. - Relevé n 12,6 ex., fauchage de Rapistrum rugosum (L.) All.

Psylliodes laticollis Kutschera, 1864 (fig. 4). - Relevé n 9, 3 ex.

Psylliodes vehemens vehemens Wollaston, 1854. - Relevé $\mathrm{n}^{\circ} 1,2$ ex.

Sphaeroderma rubidum (Graëlls, 1858). - Relevé $n^{\circ} 11,7$ ex.; relevé $n^{\circ} 12,3$ ex., fauchage de Rapistrum rugosum (L.) All. et de Poacées ; relevé n ${ }^{\circ} 3,1$ ex., sur Cynara cardunculus L.; relevé nº 14, 3 ex., sur Cynara cardunculus L.

\section{Sous-famille Chrysomelinae}

Chrysolina bankii (Fabricius, 1775). - Relevé n³, 3 ex., sous les pierres ; relevé nº 6,2 ex., sous les pierres; relevé nº 7, 1 ex., sous les pierres; relevé n 14, 6 ex., au collet de Plantago coronopus L. (sensu lato).

\section{Sous-famille Criocerinae}

Oulema duftschmidi (Redtenbacher, 1874). - Relevé ${ }^{\circ} 11,2$ ex., fauchage de Phalaris sp.; relevé ${ }^{\circ} 12$, 9 ex., fauchage de Phalaris sp. 

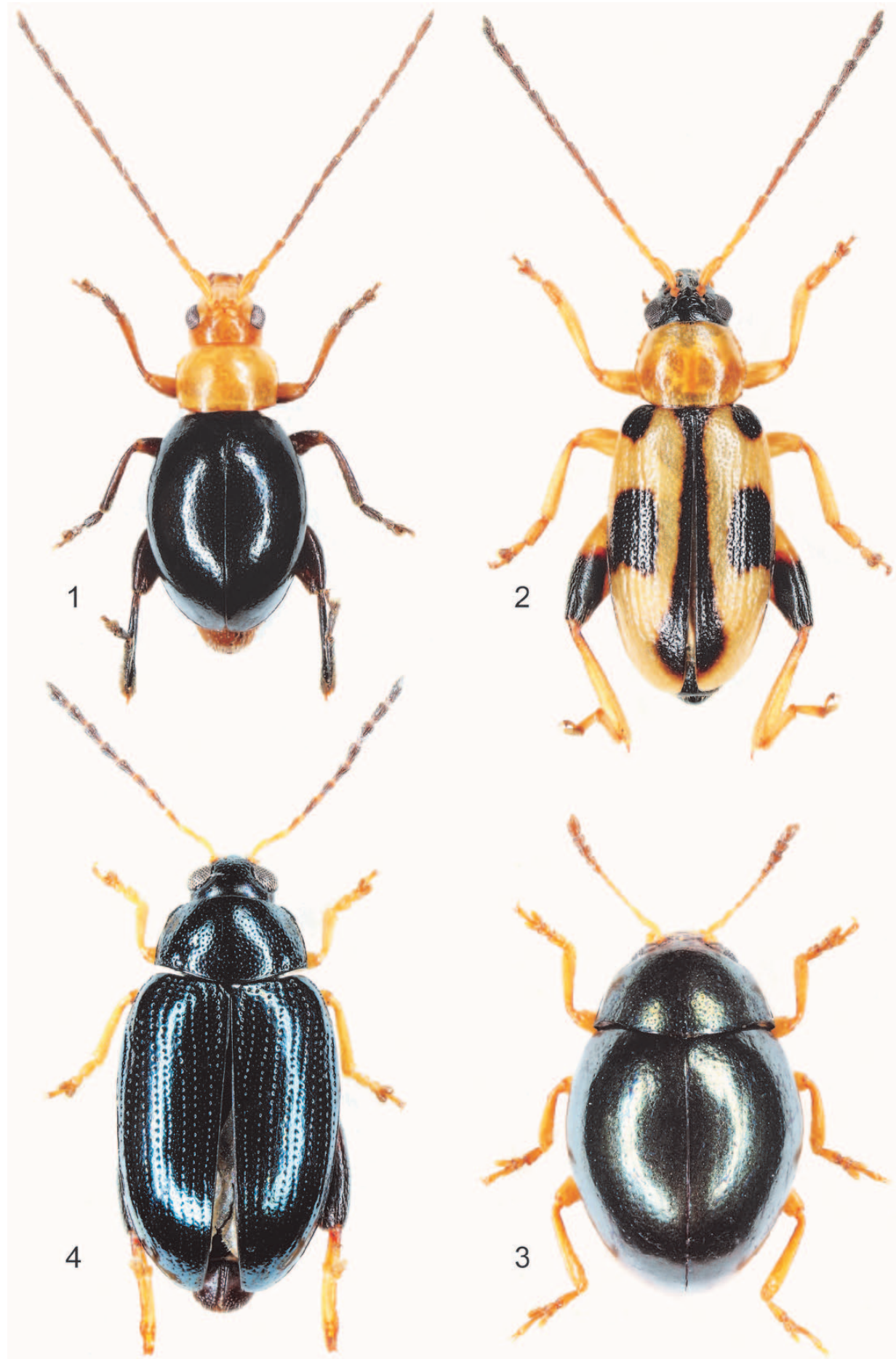

Fig. 1-4. - 1, Longitarsus cinerariae Wollaston (longueur : $3,2 \mathrm{~mm}$ ). - 2, Longitarsus isoplexidis Wollaston (longueur : 3,6 mm). - 3, Mniophilosoma laeve Wollaston (longueur : 1,9 mm). - 4, Psylliodes laticollis Kutschera (longueur : $3,3 \mathrm{~mm})$. (Clichés Ph. Ponel). 


\section{Sous-famille Cryptocephalinae}

Cryptocephalus crenatus Wollaston, 1854 . - Relevé n³, 1 ex., battage.

\section{Discussion}

Les deux sessions de prospection dans l'archipel de Madère ont permis de collecter 15 espèces de Chrysomelidae. Parmi ces espèces, la grande majorité (12 espèces) appartient à la sousfamille des Alticinae. Les Chrysomelinae, Criocerinae et Cryptocephalinae sont respectivement représentés dans nos relevés par une seule espèce. Quatre espèces méritent un commentaire particulier.

Longitarsus melanocephalus (Degeer, 1775). - Cette Altise (8 ex.) a été collectée le 1.IV.2015 sur l'île de Madère, au sud de Santana au niveau d'une friche en bord de route (altitude : $580 \mathrm{~m})(\mathrm{JDCV})$. Les conditions précises des captures, effectuées au filet-fauchoir, n'ont malheureusement pas été notées. Cette espèce n'était pas connue de l'archipel de Madère. Il s'agit d'une Altise à large répartition paléarctique associée à diverses espèces de Plantago (DoguET, 1994). Elle a peut-être été introduite accidentellement à Madère.

Mniophilosoma laeve Wollaston, 1854 (fig. 3). - D'après ERBER \& FrAnQuinho Aguiar (1996), M. laeve est largement répartie sur l'île de Madère, et se trouve souvent par exemplaires isolés ou en petit nombre. L'espèce se développerait aux dépens de Lichens. Ces mœurs inhabituelles pour une Altise expliquent probablement la rareté apparente de cette espèce. Nous l'avons découverte dans la laurisylve, dans deux localités : Encumeada, Levada do Norte (913 m), 2 ex. le 8.VII.2001 (PP) et Fanal, Casa do Guarda Florestal (1161 m), 1 ex. le 13.VII.2001 (PP). Tous

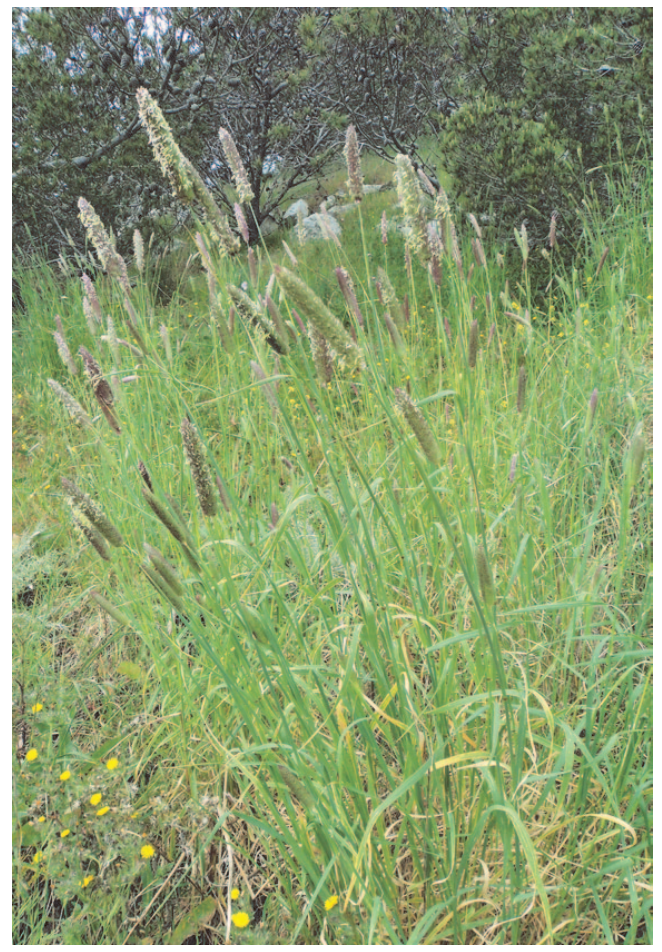

Fig. 5. - Phalaris sp., végétal sur lequel se trouvait Oulema duftschmidi (île de Porto Santo). (Cliché J.-D. ChapelinViscardi). les spécimens ont été capturés par battage de troncs et branches de vieux arbres couverts de Mousses et de Lichens.

Psylliodes laticollis Kutschera, 1864 (fig. 4). - P. laticollis a été détectée à São Jorge, au niveau d'une falaise littorale (18 m), 3 ex., le 9.VII.2001 (PP). La plante-hôte n'a pas été notée mais ces trois exemplaires ont été capturés par battage de plantes rudérales dans un milieu très anthropisé. Cette espèce n'a été découverte sur l'île qu'en 1985 (ERBER, 1986); elle n'était connue que d'un très petit nombre de localités. ERBER (1986) l'a observée sur Brassica oleracea L. Doguet (1994) la cite sur Nasturtium officinale L. Cette dernière plante-hôte semble peu probable pour notre station de São Jorge, qui ne paraît pas favorable à cette plante très hygrophile.

Oulema duftschmidi (Redtenbacher, 1874). - Nous avons relevé cette espèce le 4.IV.2015 sur l'île de Porto Santo (JDCV). Deux spécimens ont été trouvés au nord du Pico Castelo dans une zone forestière (environ $300 \mathrm{~m}$ ) et neuf spécimens au sud du Pico do Facho (environ $450 \mathrm{~m}$ ). Tous les spécimens ont été récoltés par fauchage de Phalaris sp. (fig. 5). 
D'après le catalogue de Borges et al. (2008), la seule espèce du genre connue de l'archipel est Oulema melanopus (Linné, 1761). Pourtant, O. duftschmidi avait été signalée des archipels de Madère et des Canaries par BERTi (1989), après examens des spécimens contenus dans les collections du Muséum national d'Histoire naturelle à Paris. L'étude de matériel par BEZDEK \& BASELga (2015), dans le cadre d'une révision générique ouest-paléarctique, ainsi que nos relevés, confirment la présence de $O$. duftschmidi dans l'archipel.

Actuellement et à notre connaissance, aucun spécimen de $O$. melanopus identifié d'après l'étude des pièces sclérifiées du sac interne de l'édéage n'a été signalé de Madère. BEzDEK \& Baselga (2015) indiquent que les signalements anciens (Wollaston, 1854) pourraient être à attribuer à $O$. duftschmidi du fait de la délicate séparation entre les deux espèces jumelles.

\section{Conclusion}

Ces quelques données nouvelles montrent que, malgré sa relative pauvreté, la faune de Chrysomélides de l'archipel de Madère reste encore imparfaitement connue et que des découvertes restent à faire, comme le suggérait déjà JoLIVET (2007). Citons par exemple le cas de Chrysolina fragariae, une espèce mythique que peu d'entomologistes ont eu la chance de rencontrer, mis à part ERBER (1984a, 1984b) qui a pu réaliser de belles observations sur cette espèce si particulière. Même d'éminents spécialistes de la famille ne sont pas parvenus à l'observer sur le terrain (JoLIVET, 2007). Selon Erber elle serait strictement cantonnée aux environs de Ribeiro Frio, peut-être éliminée d'autres localités de l'île par les feux, l'urbanisation et les plantations d'Eucalyptus (Jolivet, 2007). Dans le cadre de la préservation du patrimoine naturel, il semble crucial d'améliorer notre connaissance de l'entomofaune, cette espèce étant le seul Coléoptère de l'archipel de Madère inscrit à la liste des 100 espèces macaronésiennes, faune et flore confondues, menacées et prioritaires en termes de gestion (AGUiAR, 2008).

Remerciements. - Merci à Henri Michaud (Conservatoire Botanique National Méditerranéen, Hyères) qui a eu l'amabilité d'identifier les plantes sur lesquelles certaines chrysomèles ont été récoltées, et à Jan Bezdek qui nous a informé sur la révision des spécimens de Oulema de Madère.

\section{Auteurs Cités}

Aguiar A., 2008. - Chrysolina fragariae Wollaston, 1854 (p. 166-167). In : Martín J. L., Arechavaleta M., Borges P. A. V. \& Faria B. (éds), Top 100. Las 100 especies amenazadas prioritarias de gestión en la región europea biogeográfia de la Macaronesia. Consejería de Medio Ambiente y Ordenación Territorial, Gobierno de Canarias.

Berti N., 1989. - Contribution à la Faune de France. L'identité d'Oulema (O.) melanopus (L.) [Col. Chrysomelidae Criocerinae]. Bulletin de la Société entomologique de France, 94 : 47-57.

Bezdek J. \& Baselga A., 2015. - Revision of western Palaearctic species of the Oulema melanopus group, with description of two new species from Europe (Coleoptera: Chrysomelidae: Criocerinae). Acta Entomologica Musei Nationalis Pragae, 55 (1) : 273-304.

Borges A. V., Abreu C., Aguiar A. M. F., Carvalho P., Jardim R., Melo I., Oliveira P., Sérgio C., Serrano A. R. M. \& VieIra P., 2008. - A list of the terrestrial fungi, flora and fauna of Madeira and Selvagens archipelagos. Secretaria Regional do Ambiente e dos Recursos Naturais do Governo Regional da Madeira, $6: 440 \mathrm{p}$.

Doguet S., 1994. - Coléoptères Chrysomelidae 2 (Alticinae). Faune de France, 80. Paris : Fédération française des Sociétés de Sciences naturelles, $694 \mathrm{p}$.

ERBER D., 1984a. - Chrysomela fragariae Woll. auf Madeira wiederentdeckt (Chrys.). Entomologische Blätter, 80 (1) : 63.

— 1984b. - Wiederfund von Chrysomela fragariae Woll. 1854 auf Madeira. Bocagiana (Museu Municipal do Funchal), 73 : 1-9. 
Erber D., 1986. - The Chrysomelidae of Madeira. Boletim do Museu Municipal do Funchal, 38 (174) : 43-69.

Erber D. \& Franguinho Aguiar A. M. F., 1996. - New and remarkable species of the coleopterous fauna of Madeira. Boletim do Museu Municipal do Fuchal, 48 (265) : 41-62.

Erber D. \& Hinterseher W., 1988. - Contribution to the knowledge of the Madeira beetles. Boletim do Museu Municipal do Fuchal, 40 (202) : 139-214.

Jolivet P., 2007. - Sur les traces de Wollaston et... bredouille. Le Coléoptériste, 10 (2) : 131-132.

Wollaston T. V., 1854. - Insecta maderensia; being an account of the insects of the islands of the Madeiran group. London: John van Voorst, XLIII +634 p. + XIII pl. 